\title{
TO DETERMINE THE FREQUENCY OF UNDIAGNOSED HYPERTHYROIDISM IN PATIENTS PRESENTING WITH GENERALIZED ANXIETY DISORDER
}

Shahid Abbas

1. Professor. Department of General Medicine, Sri Aurobindo Institute of Medical Sciences \& PG Institute, Indore, Madhya Pradesh.

\section{CORRESPONDING AUTHOR}

Dr. Shahid Abbas,

21, Babji Nagar,

Behind Sagar automobiles near Shalimar township,

A. B. Road, near Dewas Naka. Indore (M.P.).

E-mail: drshahidabbas786@gmail.com

Ph: 00919993986152

ABSTRACT: OBJECTIVE: To determine the frequency of hyperthyroidism in patients presenting with generalized anxiety disorder. MATERIAL AND METHODS: This prospective analytical study was carried out on 150 patients presenting with Generalized Anxiety disorder following the criteria of DSM-IV-TR presenting to the Medical OPD of Sri Aurobindo Institute of Medical Sciences, Indore (M.P) from Jan 2011 to Nov 2012 with symptoms of anxiety, worry, palpitations, sweating, tremors, anorexia, hot flushes, restlessness, difficulty in concentrating and weight loss and those experiencing periods of intense fear were enrolled. All those patients who had been diagnosed or were being treated for thyroid disease or had undergone thyroid surgery or radioablation of thyroid gland were excluded. In addition patients who had anxiety with concomitant medical disease as tuberculosis, hepatitis, diabetes or hypertension etc and those on anti-arrhythmic drug amiodarone and interferon therapy for hepatitis were also excluded from the study. After a detailed history and clinical examination, thyroid function tests, E.C.G was performed in all patients and those found thyrotoxic were subjected to thyroid scan and Thyroid Peroxidase antibodies (TPOAb) test to know cause of thyrotoxicosis. Results: Out of 150 patients, $108(72 \%)$ were females and 42 (28\%) were males. They were between 18 to 48 years of age. Mean age was $28.5 \pm 4.2$ years. Out of 150 patients, 19 patients $(12.7 \%)$ were found to have elevated total and unbound thyroid hormone and low thyroid stimulating hormone levels and the rest 131 (87.3\%) patients had normal thyroid function test. All of 19 thyrotoxic patients ( 15 females and 4 males) in thyroid scan showed increased tracer uptake. Out of 19 thyrotoxic patients six had Graves' disease, ten had toxic multinodular goiter and three had toxic thyroid adenoma. CONCLUSION: Thyrotoxicosis is a common cause of generalized anxiety, patients are misdiagnosed and are being treated with anxiolytics and antidepressants, so it is very important for the physician to explore fully and give early the tests for thyroid dysfunction if required, which today are relatively simple before the diagnosis of anxiety is made and treatment started.

KEY WORDS: Thyrotoxicosis, Generalized Anxiety Disorder, DSM-IV-TR. 
INTRODUCTION: Hyperthyroidism affects $3 \%$ of women and $0.3 \%$ of males $(1)$ and is associated with significant morbidity and mortality, mainly from cardiovascular and cerebrovascular disease. ${ }^{(2,3)}$ In iodine-replete areas, the underlying diagnosis is Graves' disease in $60-80 \%$ and toxic nodular hyperthyroidism in most others. $(4,5)$ Patients with an overactive thyroid may exhibit varied signs and symptoms like marked anxiety, tension, heart palpitations, shortness of breath, chest pain, hand tremors emotional lability, impatience and irritability, distractible overactivity, exaggerated sensitivity to noise, fatigue, weight fluctuation, muscle weakness and sleeplessness, fluctuating depression with sadness, menstrual irregularities and decreased libido which are almost identical to those of anxiety and depression. In extreme cases, they may appear schizophrenic, losing touch with reality and becoming delirious or hallucinating. Pseudo-psychiatric symptoms are reported as the first sign of hyperthyroidism in somewhere between $2 \%$ and $12 \%$ of cases. Patients presenting with these symptoms are usually treated for anxiety with anti-depressants. Thyroid nodules occur in $4-15 \%$ of adult population and can be found in $90 \%$ of women over the age of sixty year.(6) Features affecting the clinical presentation are the severity of thyrotoxicosis, duration of disease, individual susceptibility to excess thyroid hormones and the patients age. Overall risk factors include an iodine rich diet, Type 1 Diabetes, pernicious anaemia, primary adrenal insufficiency, a history of autoimmune disease, or additional unrelated thyroid issues. Pregnancy induces a pseudo-hyperthyroid state through a naturally occurring increase of the T3 and T4 hormones. In the majority of women, this corrects itself within 1 to 4 months after pregnancy but in some women, however, the T3 and T4 hormones do not return to their normal levels, and the result is an ongoing hyperthyroid condition, which can very often be mistaken for post-partum depression. ${ }^{(7)}$

The most common cardiovascular manifestation is sinus tachycardia, often associated with palpitations, occasionally caused by supraventricular tachycardia. Atrial fibrillation is more common in patients $>50$ years.(7) In Grave's disease, the thyroid is usually firm and diffusely enlarged to two to three times its normal size with increased vascularity which may result in a thrill or bruit over it.(8) Patients with Grave's thyrotoxicosis lose weight despite increased appetite and food intake, thus suggesting a disturbed balance between energy intake and expenditure. Sympathetic over activity results in lid retraction, causing a staring appearance, and can occur in any form of thyrotoxicosis. Grave's disease is associated with specific eye signs that comprise Grave's ophthalmopathy. ${ }^{(8)}$

Anxiety disorders are present in 15 to $20 \%$ of medical clinic patients. Anxiety, defined as a subjective sense of unease, dread or foreboding can indicate a primary psychiatric condition or can be a component of or reaction to, a primary medical disease. (9) Approximately one third of patients presenting with anxiety have a medical aetiology for their psychiatric symptoms. Common symptoms include palpitations, sweating, tremors, shortness of breath and gastrointestinal distress.

Thyrotoxicosis is a common cause of generalised anxiety and patients are misdiagnosed and are being treated with anxiolytics, so it is very important for the physician to explore fully and give early the tests for thyroid dysfunction if required, which today are relatively simple before the diagnosis of anxiety is made and treatment started. So the aim of this study was to determine the number of patients having undiagnosed thyrotoxicosis, who are being newly diagnosed cases of generalized anxiety disorder. 
MATERIAL AND METHODS: This prospective analytical study was carried out on one hundred and fifty consecutive patients with Generalized Anxiety disorder following the criteria of DSM IV TR presenting to the Medical OPD of Sri Aurobindo Institute of Medical Sciences Indore (M.P) from Jan 2011 to Nov 2012 with symptoms of excessive anxiety and worry, palpitations, sweating, tremors, anorexia, hot flushes, restlessness, inability to relax, difficulty in concentrating and weight loss and those experiencing periods of intense fear were enrolled.

Careful history was taken and detailed physical examination was done. The clinical parameters used for thyrotoxicosis were tremors, anxiety, palpitations, sweating, a resting pulse above 90 beats per minute, weight loss, a palpable thyroid gland and thyroid eye signs as lid lag, proptosis or ophthalmoplegia. This is a purposive study following the criteria of DSM-IV-TR.(10) According to DSM-IV-TR (the American Psychiatric Association Classification Book) a mental disorder due to a general medical condition characterized by the presence of mental symptoms that are judged to be the direct psychological consequence of general medical condition-in this case thyrotoxicosis. Purposive sampling targets a group of people like with generalized anxiety disorder.

DSM-IV-TR diagnostic criteria for generalized anxiety disorder are as follows: A. Excessive anxiety and worry (apprehensive expectation), occurring more-days-than-not for at least 6 months, about a number of events or activities (such as work or school performance).

B. The person finds it difficult to control the worry. C. The anxiety and worry are associated with three (or more) of the following six symptoms (with at least some symptoms present for more-days-than-not for the past 6 months).

1. restlessness or feeling keyed up or on edge

2. being easily fatigued

3. difficulty concentrating or mind going blank

4. irritability

5. muscle tension

6. sleep disturbance (difficulty falling or staying asleep or restless unsatisfying sleep)

D. The focus of the anxiety and worry is not confined to disorders such as social phobia, obsessive compulsive disorder (OCD), post traumatic stress disorder (PTSD) etc. E. The anxiety, worry, or physical symptoms cause clinically significant distress or impairment in social, occupational, or other important areas of functioning F. The disturbance is not due to the direct physiological effects of a substance (e.g., a drug of abuse, a medication) or a general medical condition (e.g., hyperthyroidism), and does not occur exclusively during a mood disorder, psychotic disorder, or a pervasive developmental disorder.

These patients were evaluated with a detailed history, with an effort to determine whether the anxiety antedates or postdates a medical illness or is due to medication side effect. After history and thorough physical examination, all patients underwent thyroid function test. The serum T3, T4 and TSH were estimated by ELISA method to group them as normal, hypothyroid and hyperthyroid subjects and after being diagnosed as having hyperthyroidism they were subjected to thyroid scan and Thyroid Peroxidase antibodies (TPOAb) test to know cause of thyrotoxicosis. Electrocardiogram with BPL Cardiart 6108 T ECG Machine was also performed in all patients. 
EXCLUSION CRITERIA: All those patients who had diagnosed thyroid disease, were being treated for thyroid disease or had undergone thyroid surgery or radioablation of thyroid gland were excluded from the study, patients with neurological disorder, prior psychiatric disorder, other endocrine disorders, substance abuse and who had anxiety with concomitant medical disease, such as tuberculosis, hepatitis, diabetes or hypertension etc and those on antiarrhythmic drug amiodarone and interferon therapy for hepatitis were also excluded from the study.

RESULTS: A total of one hundred and fifty patients diagnosed as having generalized anxiety disorder were enrolled in the study. They were between 18 to 48 years of age. Mean age was $28.5 \pm 4.2$ years. One hundred and eight $(72 \%)$ out of the total were females and fourty two (28\%) were males (Table-1). Out of one hundred and fifty patients, nineteen patients $(12.7 \%)$ were found to have elevated total and unbound thyroid hormone and low thyroid stimulating hormone levels. One hundred and thirty one $(87.3 \%)$ patients had normal thyroid function test (Table-2). Out of 19 thyrotoxic patients 15 were females and 4 were males (Table-3). Thyroid scan showed that in our study all thyrotoxic patients $(12.7 \%)$ had increased tracer uptake. TPOAb test and uniform tracer uptake on a thyroid scan was positive in six patients (five female and one male) suggesting Graves' disease. Patchy distribution on thyroid scan suggesting toxic multinodular goiter (MNG) was found in 10 thyrotoxic patients (eight females and two males) and unifocal activity corresponding to a nodule with suppression of rest of the thyroid gland suggesting a toxic adenoma was found in three patients (two female and one male) (Table-4).

Twelve patients had sinus tachycardia, four patients had premature atrial beats, two had paroxysmal supraventricular tachycardia and one had atrial fibrillation on electrocardiogram (ECG). Rest of the ECGs were normal.

DISCUSSION: Alexander considered that thyrotoxicosis was a psychosomatic disorder, but this idea was not supported by the evidence.(1) However there are reports suggesting that stressful life events may precipitate the onset of disease. In our study we could not clearly relate between stress and the thyroid. The number of people who cite unusually stressful experiences before the onset of hyperthyroidism seems to bear out the theory of stress as a precipitating factor. ${ }^{(11)}$ While others can come through the same upheavals without developing thyroid disease, some perhaps are predisposed to it. On the other hand, it can be argued that the illness itself, before its symptoms are manifested, is contributing to the situation of stress.

Thyrotoxicosis is defined as a state of thyroid hormone excess and the major etiologies are Grave's disease, toxic multinodular goiter and toxic adenomas. Clinical features of thyrotoxicosis can mimic certain aspects of mental disorders as panic attacks and generalized anxiety disorder.(12-14) Older studies were mainly concerned with the prevalence of psychiatric disorders but antedated the general use of systematic diagnostic criteria concerning both hyperthyroidism and mental functioning. More recent studies have usually used such criteria for qualitative evaluation of mental state to allow specific psychiatric diagnoses to be made, they have also used psychometric rating scales that quantify psychological symptoms and functioning.

Using standard psychometric rating scales and neuropsychological tests, several studies have investigated mental symptoms and psychological functioning in patients with hyperthyroidism, most, but not all, of whom have had Graves' disease. 
Like our study previous studies have demonstrated that patients with overt hyperthyroidism are more likely to have symptoms of depression and anxiety than are healthy subjects. ${ }^{(15-18)}$ and physical symptoms such as palpitations, increased heart rate, excessive sweating and tremor may reduce quality of life.(19-21)

An early study (22) reported an association between scores on tests of anxiety or depression and serum concentrations of thyroid hormones in patients with Graves' hyperthyroidism. Kathol and Delahunt(23) reported that approximately one-third of 32 patients with hyperthyroidism (caused by Graves' disease in 28 of these patients) met DSM-III(24) criteria for depression and approximately one-half met the criteria for anxiety disorders. Applying Research Diagnostic Criteria,(25) Trzepacz et al.(26) studied 13 patients with hyperthyroidism caused by Graves' disease and reported that every patient met the criteria for at least one psychiatric diagnosis (most often depression, anxiety or hypomania). Placidi et al.(27) studied 93 patients with hyperthyroidism using DSM-III-R(28) criteria. One- third of their patients had generalised anxiety disorder (GAD). Brownlie et al.(29) retrospectively studied 18 patients with comorbid newly diagnosed hyperthyroidism and psychoses. In 11 younger patients, hyperthyroidism was caused by diffuse goiter (suggesting Graves' disease) and in seven older patients by nodular goiter. Seven patients met ICD-9(30) criteria for mania (five with diffuse goiter, two with nodular goiter); seven patients met such criteria for depression (four with diffuse goiter, three with nodular goiter); two patients met the criteria for schizophreniform psychosis (one with diffuse goiter and one with nodular goiter); one met the criteria for paranoid psychoses (with diffuse goiter); and one patient met the criteria for delirium (with nodular goiter).

Recently, Bunevicius et al.(31) studied 30 women treated for both Graves' hyperthyroidism and Graves' ophthalmopathy, and 45 women with gynaecological disorders who had normal thyroid function. The women with persistent Graves' hyperthyroidism had a higher prevalence of current DSM- IV/ICD-10 mood and anxiety disorders and a higher prevalence of past episodes of mania or hypomania compared with the control women. The women with Graves' disease that was in remission also had more past episodes of mania or hypomania than the control women.

The symptoms of generalized anxiety disorder (GAD) are persistent and not restricted to any particular set of circumstances. The lifetime prevalence of GAD is 4.1 to $6.6 \%$, ${ }^{(32)}$ which is higher than that of the other anxiety disorders. The prevalence of GAD in patients visiting physicians' offices is twice that found in the community. ${ }^{(33)}$ It is more prevalent in women than in men (in our study female : male ratio is 2.57: 1 ) with the median age of onset occurring during the early 20 s. $^{(34)}$ The onset of symptoms is usually gradual, although GAD can be precipitated by stressful life events. The condition tends to be chronic with periods of exacerbation and remission. ${ }^{34)}$

Palpitations, caused by sinus tachycardia and occasionally by atrial fibrillation, are the most frequent cardiovascular symptom of thyrotoxicosis,(32) and are usually regarded as the part of generalized anxiety disorder. Thyroid hormone excess should routinely be excluded in patients with atrial fibrillation. There are always some psychological symptoms in hyperthyroidism, including restlessness, irritability and distractibility, which may be so marked as to resemble anxiety disorder. The severity of emotional symptoms are not related to thyroid hormone levels but they usually improve following antithyroid therapy.(7)

This study was conducted on one hundred and fifty patients diagnosed as having generalised anxiety disorder attending Medical OPD more in women than in men. Out of 150 
patients 19 patients $(12.7 \%)$ were found to have elevated total and unbound thyroid hormone. Out of 19 patients found thyrotoxic 15 were female and 4 were male.

This study stresses the importance of exclusion of thyrotoxicosis, presenting with anxiety as the chief complaint. The most discriminating signs of thyrotoxicosis are a palpable thyroid, a sleeping pulse above 90 beats per minutes, atrial fibrillation and tremors. ${ }^{(18)}$ The most discriminating signs of thyrotoxicosis in our study were tachycardia above 95 per minute, sweating, a palpable thyroid gland and wasting. For this reason a thorough history, physical examination and judicious use of laboratory tests are required. Prompt diagnosis is essential to maximize the clinical outcome.(19) Primary care physicians should base referrals to a psychiatrist on the basis of signs and symptoms of a mental disorder and absence of organic disease.

CONCLUSION: Detection of the thyroid problem is complicated by the fact that everyone feels anxiety and tension to some degree, that many thyroid symptoms are similar to those of other diseases. Disturbance of thyroid function may be accompanied by psychological disturbances which can mimic other psychiatric syndromes. But the results of overlooking the thyroid can be serious. It is very important for the physician to explore fully and give early the tests for thyroid dysfunction if required, which today are relatively simple. When effective thyroid treatment is begun, the general response is quite favourable. Vitality returns and the mental processes become efficient again. If there is a residue of emotional difficulties, it may be related not to the thyroid gland but to other aspects of life.

\section{REFERENCES:}

1. Tunbridge WM, Evered DC, Hall R, Appleton B, Brewis M, Clark F, Evans JG, Young E, Bird T, Smith PA 1977. The spectrum of thyroid disease in a community: the Whickham survey. Clin Endocrinol (Oxf) 7:481- 493.

2. Franklyn JA, Sheppard MC, Maisonneuve P. Thyroid function and mortality in patients Thyroid Function and Mortality in Patients Treated for Hyperthyroidism. JAMA. 2005;294(1):71-80.

3. Klein I, Ojamaa K. Thyroid hormone and the cardiovascular system. N Engl J Med. 2001;344501- 509.

4. Cooper DS. Hyperthyroidism. Lancet. 2003 Aug 9;362(9382):459-68.

5. Weetman AP .Graves' disease. N Engl J Med 2000; 343:1236-1248.

6. Sachmechi I. Thyroid carcinoma in single cold nodule and in cold nodules of multinodular goitres. Endocr Pract 2000; 6:5-7.

7. Jameson JL. Disorders of the thyroid gland. In: Kasper DL, Braunwald E, Fauci AS, Hauser, Longo DL, Jameson JL, et al. Eds Harrison's principles of internal medicine.18th ed Vol. II, McGraw-Hills, NY, p 2911-2939.

8. Streetman DD, Khanderia U. Diagnosis and treatment of Graves disease. Ann Pharmacother. 2003 Jul-Aug;37(7-8):1100-9.

9. Denicoff J. Neuropsychiatric manifestation of altered thyroid state. Am J Psychiat 1990; 147:94-98.

10. American Psychiatric Association. Diagnostic and Statistical Manual of Mental Disorders, Fourth Edition Revised. Text revision. Psychiatric Press, Inc., Washington, DC: 2000.

11. Topcu CB, Celik O, Tasan E. Effect of stressful life events on the initiation of graves' disease. Int J Psychiatry Clin Pract. 2012 Oct;16(4):307-11. 
12. Gelder M G, Juan J. Lopez-Ibor, Andrerson N. Editors Oxford textbook of Psychiatry, 3rd E d. Oxford University Press, p 162-4.

13. Larisch R, Kley K, Nikolaus S, et al. Depression and anxiety in different thyroid function states. Horm Metab Res 2004 Sep; 36 (9): 650-3.

14. Schlote B, Nowotny B, Schaaf L, et al. Subclinical hyperthyroidism: physical and mental state of patients. Eur Arch Psychiatry Clin Neurosci 1992; 241 (6): 357-64.

15. Lacovides A, Fountoulakis KN, Grammaticos P, et al. Difference in symptom profile between generalized anxiety disorder and anxiety secondary to hyperthyroidism. Int J Psychiatry Med 2000; 30 (1): 71-81.

16. Demet MM, Ozmen B, Deveci A, et al. Depression and anxiety in hyperthyroidism. Arch Med Res 2002 Nov-Dec; 33 (6): 552-6.

17. Lee IT, Sheu WH, Liau YJ, et al. Relationship of stressful life events, anxiety and depression to hyperthyroidism in an Asian population. Horm Res 2003; 60 (5): 247-51.

18. Gulseren S, Gulseren L, Hekimsoy Z, et al. Depression, anxiety, health-related quality of life, and disability in patients with overt and subclinical thyroid dysfunction. Arch Med Res 2006 Jan; 37 (1): 133-9.

19. Rockel M, Teuber J, Schmidt R, et al. Correlation of "latent hyperthyroidism" with psychological and somatic changes [in German]. Klin Wochenschr 1987 Mar 16; 65 (6): 264-73.

20. Biondi B, Palmieri EA, Fazio S, et al. Endogenous subclinical hyperthyroidism affects quality of life and cardiac morphology and function in young and middle-aged patients. J Clin Endocrinol Metab 2000 Dec; 85 (12): 4701-5.

21. Biondi B, Palmieri EA, Klain M, et al. Subclinical hyperthyroidism: clinical features and treatment options. Eur J Endocrinol 2005 Jan; 152 (1): 1-9.

22. Whybrow PC, Prange Jr AJ, Treadway CR. Mental changes accompanying thyroid gland dysfunction: a reappraisal using objective psychological measurement. Arch Gen Psychiatry 1999 Jan; 20 (1): 48-63.

23. Kathol RG, Delahunt JW. The relationship of anxiety and depression to symptoms of hyperthyroidism using operational criteria. Gen Hosp Psychiatry 1986 Jan; 8 (1): 23-8.

24.. American Psychiatric Association. Diagnostic and statistical manual of mental disorders. 3rd ed. Washington, DC: American Psychiatric Association, 1980.

25. Spitzer RL, Endicott J, Robins E. Research diagnostic criteria:rationale and reliability. Arch Gen Psychiatry 1978 Jun; 35 (6): 773-82.

26. Trzepacz PT, McCue M, Klein I, et al. A psychiatric and neuropsychological study of patients with untreated Graves' disease. Gen Hosp Psychiatry 1988 Jan; 10 (1): 49-55.

27. Placidi GPA, Boldrni M, Patronelli A, et al. Prevalence of psychiatric disorders in thyroid diseased patients. Neuropsychobiology 1998 Nov; 38 (4): 222-5.

28. American Psychiatric Association. Diagnostic and statistical manual of mental disorders. 3rd rev. ed. Washington, DC: American Psychiatric Association, 1987.

29. Brownlie BEW, Rae AM, Walshe JWB, et al. Psychoses associated with thyrotoxicoses. "Thyrotoxic psychoses": a report of 18 cases, with statistical analysis of incidence. Eur J Endocrinol 2000 May; 142 (5): 438-44.

30. Manual of the international statistical classification of diseases,injuries, and causes of death. Vol.1. Geneva: World Health Organization, 1977. 
31. Bunevicius R, Velickiene D, Prange Jr AJ. Mood and anxiety disorders in women with treated hyperthyroidism and ophthalmopathy caused by Graves' disease. Gen Hosp Psychiatry 2005 Mar -Apr; 27 (2): 133-9.

32. Robins LN, Regier DA. Psychiatric disorders in America: the epidemiologic catchment area study Robins LN,. Regier DA, eds; with a foreword by Freedman DX. New York: Free Press, 1991.

33. Schweizer E, Rickels K. Strategies for treatment of generalized anxiety in the primary care setting. J Clin Psychiatry. 1997;58(suppl 3):27-33.

34. Rickels K, Schweizer E. The clinical course and long-term management of generalized anxiety disorder. J Clin Psychopharmacol. 1990;10:1015-19.

TABLE 1: SEX WISE DISTRIBUTION OF PATIENTS

\begin{tabular}{|c|c|c|}
\hline Sex & Number & \% Age \\
\hline Female & 108 & 72 \\
\hline Male & 42 & 28 \\
\hline Total & 150 & 100 \\
\hline
\end{tabular}

TABLE 2: FREQUENCY OF THYROTOXICOSIS IN PATIENTS WITH GENERALIZED ANXIETY

\begin{tabular}{|c|c|c|}
\hline Patients & Number & \% Age \\
\hline Thyrotoxic & 19 & 12.7 \\
\hline Euthyroid & 122 & 87.3 \\
\hline Total & 150 & 100 \\
\hline
\end{tabular}

TABLE 3 : SEX WISE DISTRIBUTION OF THYROTOXIC PATIENTS

\begin{tabular}{|c|c|c|}
\hline SEX & Number of Thyrotoxic Patients & $\%$ Age \\
\hline Female & 15 & 78.9 \\
\hline Male & 4 & 21.1 \\
\hline Total & 19 & 100 \\
\hline
\end{tabular}

TABLE 4 : CAUSES OF THYROTOXICOSIS

\begin{tabular}{|c|c|c|c|c|}
\hline $\begin{array}{c}\text { Causes of } \\
\text { Thyrotoxicosis }\end{array}$ & $\begin{array}{l}\text { Female } \\
(\mathrm{n}=15)\end{array}$ & $\begin{array}{l}\text { Male } \\
(\mathrm{n}=4)\end{array}$ & $\begin{array}{l}\text { Thyroidperoxidase antibody } \\
\text { (TPOAb) }\end{array}$ & $\begin{array}{l}\text { Total } \\
(n=19)\end{array}$ \\
\hline Graves Disease & 5 & 1 & + & $6(31.6 \%)$ \\
\hline $\begin{array}{c}\text { Toxic Multinodular } \\
\text { goitre }\end{array}$ & 8 & 2 & - & $\begin{array}{c}10( \\
52.6 \%)\end{array}$ \\
\hline Toxic adenoma & 2 & 1 & - & $3(15.8 \%)$ \\
\hline
\end{tabular}

